\title{
THE SUMMATION OF CERTAIN SERIES OF INFINITE REGRESSIVE ISOLS ${ }^{1}$
}

\section{F. J. SANSONE}

1. Introduction. Denote the set of all non-negative integers by $\epsilon$, the collection of all isols by $\Lambda$, and the collection of all regressive isols by $\Lambda_{R}$. If $f$ is a function, we denote the range of $f$ and domain of $f$ by $\rho f$ and $\delta f$ respectively. Dekker, in [1], defined and studied an infinite sum of non-negative integers. In this paper, we consider an infinite sum of infinite, regressive isols of the form $T-k$ for some $T \in \Lambda_{R}-\epsilon$.

2. Summary. We use the well-known pairing function $j(x, y)$ which maps $\epsilon^{2}$ one to one onto $\epsilon$ and the functions $k(z), l(z)$ such that $j(k(z), l(z))=z$. We also employ the mapping $\Phi_{f}$, introduced in [6] as well as the partial ordering $\leqq *$ of $\Lambda$, defined in [2]. For $k \in \epsilon$ and $t_{n}$ a regressive function, the set $\left(t_{k}, t_{k+1}, t_{k+2}, \cdots\right)$ is denoted by $\rho t_{n+k}$.

Definition. Let $T$ and $U$ be infinite, regressive isols and $a_{n}$ a rocursive function. Then

$$
\sum_{U}\left(T-a_{n}\right)=\operatorname{Req} \bigcup_{k=0}^{\infty} j\left(u_{k}, \rho t_{n+a(k)}\right),
$$

where $u_{k}$ and $t_{n}$ are any regressive functions ranging over sets in $U$ and $T$ respectively.

The principal results of this paper are as follows. Let $a_{n}$ be a strictly increasing, recursive function. Then for $T, U \in \Lambda_{R}-\epsilon$,

$$
\sum_{U}\left(T-a_{n}\right)=\sum_{V}\left(T-a_{n}\right), \text { where } \quad V=\min \left(\Phi_{a}(T), U\right) .
$$

Moreover, with respect to the regressive isol $V$, the sum can be distributed over the difference $T-a_{n}$.

We note here several properties of the sum. It is readily shown that if $t_{n}, t_{n}^{*}$ are any two regressive functions ranging over sets in $T$ and $u_{k}, u_{\mathrm{k}}^{*}$ are any two regressive functions ranging over sets in $U$, then

$$
\bigcup_{k=0}^{\infty} j\left(u_{k}, \rho t_{n+a(k)}\right) \simeq \bigcup_{k=0}^{\infty} j\left(u_{k}^{*}, \rho t_{n+a(k)}^{*}\right) \text {. }
$$

Presented to the Society, January 24, 1964; received by the editors June 12, 1964.

1 The results in this paper are contained in the author's doctoral dissertation written at Rutgers, the State University, under the direction of Professor J. C. E. Dekker. 
Hence the sum is uniquely defined. Moreover, the sum depends only on $U$ and the infinite sequence of isols, $\left\{T-a_{n}\right\}$, and not on the choice of $T$ itself, for it is easily proved that for $U, T \in \Lambda_{R}-\epsilon$ and $k$ any integer such that $a_{n}+k \geqq 0$ for all $n$,

$$
\sum_{U}\left((T+k)-\left(a_{n}+k\right)\right)=\sum_{U}\left(T-a_{n}\right) .
$$

It is also apparent that for $T, U \in \Lambda_{R}-\epsilon$, the sum, $\sum_{U}\left(T-a_{n}\right) \in \Lambda$.

3. Principal results. If $t_{n}$ is a regressive function having the regressing function $p(x)$, we make use of the partial recursive extension $p^{*}(x)$ of $t^{-1}$, defined by $p^{*}(x)=(\mu y)\left[p^{\nu+1}(x)=p^{y}(x)\right]$.

THEOREM 1. Let $a_{n}$ be a strictly increasing, recursive function. Then for $T, U \in \Lambda_{R}-\epsilon$,

$$
\sum_{U}\left(T-a_{n}\right)=\sum_{V}\left(T-a_{n}\right), \text { where } V=\min \left(\Phi_{a}(T), U\right) .
$$

Proof. We note that since $a_{n}$ is strictly increasing and recursive, $\Phi_{a}(T)$ is a regressive isol and hence $V$ is well defined and is also regressive. Let $t_{n}, u_{k}$ be regressive functions ranging over sets in $T$ and $U$ respectively. By definition:

$$
\begin{aligned}
& \sum_{U}\left(T-a_{n}\right)=\operatorname{Req} \bigcup_{k=0}^{\infty} j\left(u_{k}, \rho t_{n+a(k)}\right), \\
& \sum_{V}\left(T-a_{n}\right)=\operatorname{Req} \bigcup_{k=0}^{\infty} j\left(j\left(t_{a(k)}, u_{k}\right), \rho t_{n+a(k)}\right) .
\end{aligned}
$$

Denote the sets appearing on the right in (1) and (2) by $\alpha$ and $\beta$ respectively.

Let $p(x)$ and $q(x)$ be regressing functions of the regressive functions $t_{n}$ and $u_{k}$ respectively. Define

$$
f(z)=j\left[j\left(p^{p^{*} l(z)-a_{q}^{*} k(z)} l(z), k(z)\right), l(z)\right] .
$$

Let

$$
g(z)=j(l k(z), l(z)) .
$$

Clearly, both $f$ and $g$ are partial recursive functions. For $z \in \alpha$, $p^{*} l(z)$ and $a q^{*} k(z)$ are defined, and $a q^{*} k(z) \leqq p^{*} l(z)$. Hence $\alpha \subset \delta f$. To verify that $f(\alpha)=\beta$, it is sufficient to note that for $z \in \alpha$, there exists $m$ such that $k(z)=u_{m}$ and $l(z)=t_{a(m)+s}$ for some $s \in \epsilon$. Hence

$$
p^{p^{*} l(z)-a q^{*} k(z)} l(z)=t_{a(m)} .
$$


It readily follows that $f(\alpha)=\beta$. That $f$ is $1-1$ on $\alpha$ is a consequence of the fact that $j(x, y)$ is $1-1$. Clearly, $\beta \subset \delta g, g(\beta)=\alpha$ and $g$ is $1-1$ on $\beta$. Furthermore, for $z \in \alpha, g f(z)=z$. An application of Proposition 1 of [1] completes the proof.

CoROLLARY. Let $a_{n}$ be a strictly increasing, recursive function. Let $T, U, V \in \Lambda_{R}-\epsilon$. Then

$$
\left[\Phi_{a}(T) \leqq * U, \Phi_{a}(T) \leqq * V\right] \Rightarrow \sum_{U}\left(T-a_{n}\right)=\sum_{V}\left(T-a_{n}\right) .
$$

Proof. Since $\Phi_{a}(T) \leqq * U, \min \left(\Phi_{a}(T), U\right)=\Phi_{a}(T)$. Since $\Phi_{a}(T) \leqq * V$, $\min \left(\Phi_{a}(T), V\right)=\Phi_{a}(T)$. The result follows by applying the theorem to both sums.

THEOREM 2. Let $a_{n}$ be a strictly increasing, recursive function. Then for $T, U \in \Lambda_{R}-\epsilon$,

$$
\sum_{V}\left(T-a_{n}\right)=T V-\sum_{V} a_{n}, \text { where } V=\min \left(\Phi_{a}(T), U\right) .
$$

Proof. It suffices to prove

$$
\sum_{V}\left(T-a_{n}\right)+\sum_{V} a_{n}=V T .
$$

Let $t_{n}$ and $u_{k}$ be regressive functions ranging over sets in $T$ and $U$ respectively. Let

$$
\begin{aligned}
& \alpha=\bigcup_{k=0}^{\infty} j\left[j\left(t_{a(k)}, u_{k}\right), \rho t_{n+a(k)}\right], \\
& \beta=\bigcup_{k=0}^{\infty} j\left[j\left(t_{a(k)}, u_{k}\right), \nu\left(a_{k}\right)\right], \\
& \gamma=j\left[\rho j\left(t_{a(k)}, u_{k}\right), \rho t_{n}\right], \\
& \delta=\bigcup_{k=0}^{\infty} j\left[j\left(t_{a(k)}, u_{k}\right), t \nu\left(a_{k}\right)\right] .
\end{aligned}
$$

Here, $\nu\left(a_{k}\right)$ denotes the set $\left(0,1, \cdots, a_{k}-1\right)$. By definition, we have:

$$
\begin{aligned}
\sum_{V}\left(T-a_{n}\right) & =\operatorname{Req} \alpha, \\
\sum_{\nabla} a_{n} & =\operatorname{Req} \beta, \\
V T & =\operatorname{Req} \gamma .
\end{aligned}
$$


Since $a_{n}$ is recursive, $\alpha \mid \delta$. We also have $\alpha+\delta=\gamma$. Hence to prove (1), it suffices to show that $\beta \simeq \delta$. Let $p(x)$ be a regressing function of the regressive function $t_{n}$ and let $p^{*}(x)$ be related to $p(x)$ in the usual manner. Define

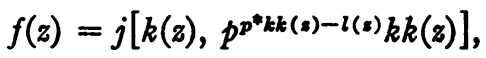

$$
\begin{aligned}
& g(z)=j\left(k(z), p^{*} l(z)\right) .
\end{aligned}
$$

Clearly, both $f$ and $g$ are partial recursive functions. Since for $z \in \beta$, $p^{*} k k(z)-l(z)$ is defined and $k k(z) \in \rho t_{n}$, we have $\beta \subset \delta f$. For $z \in \beta$, there exists $m$ such that $k k(z)=t_{a(m)}$ and $l(z)=a_{m}-(s+1)$ for some $s \in \nu\left(a_{m}\right)$. Hence

$$
p^{p^{*} k k(z)-l(z)}(k k(z))=p^{a(m)-a(m)+s+1}\left(t_{a(m)}\right)=t_{a(m)-(o+1)}
$$

and $f(\beta)=\delta$. Clearly $f$ is $1-1$ on $\beta$. The function $g(z)$ obviously has the properties:

$$
\delta \subset \delta g, \quad g(\delta)=\beta, \text { and } g \text { is } 1-1 \text { on } \delta .
$$

Since for $z \in \beta, g f(z)=z$, we have $\beta \simeq \delta$.

Combining the two preceding theorems, we obtain:

THEOREM 3. Let $a_{n}$ be a strictly increasing recursive function. Then for $T, U \in \Lambda_{R}-\epsilon$,

$$
\sum_{U}\left(T-a_{n}\right)=T V-\sum_{V} a_{n}, \text { where } V=\min \left(\Phi_{a}(T), U\right) .
$$

The following are immediate corollaries of Theorem 3.

COROLLARY 1. Let $a_{n}$ be a strictly increasing, recursive function. Let $T, U \in \Lambda_{R}-\epsilon$. Then

$$
U \leqq * \Phi_{a}(T) \Rightarrow \sum_{U}\left(T-a_{n}\right)=T U-\sum_{U} a_{n}
$$

COROLlaRY 2. Let $a_{n}$ be a strictly increasing, recursive function. Let $T, U \in \Lambda_{R}-\epsilon$. Then

$$
\Phi_{a}(T) \leqq * U \Rightarrow \sum_{U}\left(T-a_{n}\right)=T \Phi_{a}(T)-\sum_{\Phi_{a}(T)} a_{n} .
$$

COROLLARY 3. Let $a_{n}$ be a strictly increasing, recursive function and let $T \in \Lambda_{R}-\epsilon$. Then

$$
\sum_{T}\left(T-a_{n}\right)=T \Phi_{a}(T)-\sum_{\Phi_{a}(T)} a_{n}
$$


4. REMaRKs. We state here several other results whose hypotheses are more restrictive than those of Theorems 1 and 2. Their proofs will be omitted.

TheOREM 4. Let $T, U \in \Lambda_{R}-\epsilon$. If $a_{n}$ is a recursive function such that $(\forall n)\left[a_{n} \leqq n+1\right]$, then

$$
U \leqq * T \Rightarrow \sum_{U}\left(T-a_{n}\right)=T U-\sum_{U} a_{n}
$$

CoROLlaRy 1. Let $T \in \Lambda_{R}-\epsilon$. If $a_{n}$ is a recursive function satisfying the hypothesis of Theorem 4, then

$$
\sum_{T}\left(T-a_{n}\right)=T^{2}-\sum_{T} a_{n}
$$

Corollary 2. Let $T \in \Lambda_{R}-\epsilon$. Then

$$
\sum_{T}: T+(T-1)+(T-2)+\cdots=\sum_{T}: 1+2+3+\cdots
$$

The second corollary can also be obtained by an application of Theorem 3.

For every increasing, unbounded, recursive function $a_{n}$, we define

$$
\bar{a}_{n}=(\mu y)\left[a_{y}>n\right] \text {. }
$$

The function $\bar{a}_{n}$ is clearly partial recursive. Moreover, since $a_{n}$ is unbounded, it follows that $\tilde{a}_{n}$ is everywhere defined and hence recursive.

THEOREM 5. Let $a_{n}$ be an increasing, recursive function such that $(\forall n)\left[a_{n} \geqq n\right]$. Then for $T, U \in \Lambda_{R}-\epsilon$,

$$
T \leqq * U \Rightarrow \sum_{U}\left(T-a_{n}\right)=\sum_{T} \bar{a}_{n} .
$$

COROLlaRY. Let $a_{n}$ be an increasing, recursive function such that $\forall n)\left[a_{n} \geqq n\right]$. Then for $T \in \Lambda_{R}-\epsilon$,

$$
\sum_{T}\left(T-a_{n}\right)=\sum_{T} \bar{a}_{n}
$$

Results similar to those in Theorems 1 and 2 can be obtained for sums whose terms consist of a product of factors. For $T, U, V \in \Lambda_{R}-\epsilon$ and $a_{n}, b_{n}$ recursive functions, we define 


$$
\sum_{V}\left(T-a_{n}\right)\left(U-b_{n}\right)=\operatorname{Req} \bigcup_{k=0}^{\infty} j\left(v_{k}, j\left(\rho t_{n+a(k)}, \rho u_{n+b(k)}\right)\right),
$$

where $t, u, v$ are regressive functions ranging over sets in $T, U, V$ respectively.

THEOREM 6. Let $a_{n}$ and $b_{n}$ be strictly increasing, recursive functions. Let $T, U, V \in \Lambda_{R}-\epsilon$ and $M=\min \left(\Phi_{a}(T), \Phi_{b}(U), V\right)$. Then

$$
\sum_{V}\left(T-a_{n}\right)\left(U-b_{n}\right)=\sum_{M}\left(T-a_{n}\right)\left(U-b_{n}\right) .
$$

Morever, with respect to the isol $M$, the sum can be distributed over the product to obtain $M T U-T \sum_{M} b_{n}-U \sum_{M} a_{n}+\sum_{M} a_{n} b_{n}$.

\section{REFERENCES}

1. J. C. E. Dekker, Infinite series of isols, Recursive function theory, Proc. Sympos. Pure Math. Vol. 5, Amer. Math. Soc., Providence, R. I., 1962; pp. 77-96.

2. - The minimum of two regressive isols, Math. Z. 83 (1964), 345-366.

3. J. C. E. Dekker and J. Myhill, Recursive equivalence types, Univ. of Calif. Press, Berkeley, Calif., 1960.

4. A. Nerode, Extensions to isols, Ann. of Math. 73 (1961), 362-403.

5. - Extensions to isolic integers, Ann. of Math. 75 (1962), 419-448.

6. F. J. Sansone, Combinatorial functions and regressive isols, Pacific J. Math. 13 (1963), 703-707.

Case Institute of Technology 